\title{
Variables predictoras de la ansiedad en atletas veteranos españoles Predictors variables of anxiety in the Spanish master athletes
}

\author{
Antonio Zarauz Sancho, Francisco Ruiz Juan \\ Universidad de Murcia
}

\begin{abstract}
Resumen: En los últimos años se ha producido en España un incremento muy significativo de adultos mayores de 35 años que frecuentemente entrenan y compiten a todos los niveles en pistas de atletismo. Son los atletas veteranos y, sobre ellos, no hay ningún estudio a nivel nacional y son muy pocos los que hay a nivel internacional. Por ello, el objetivo de la presente investigación será; por un lado, conocer sus hábitos de entrenamiento, historial atlético y nivel de ansiedad, analizando las diferencias por sexo; por otro, obtener unos modelos predictivos de su ansiedad en función de sus hábitos de entrenamiento e historial atlético. Se parte de la hipótesis de que, en los atletas veteranos españoles, en ambos sexos, debe existir una moderada ansiedad precompetitiva (cognitiva y somática) y alta autoconfianza, y de que la ansiedad se podrá predecir por puntuar alto en el otro estado de ansiedad, por puntuar bajo en autoconfianza, así como por entrenar menos días a la semana y llevar menos años entrenando, por el mayor nivel de la competición en la que participa y por no tener apoyo de un entrenador. Por el contrario, la autoconfianza se podrá predecir por puntuar bajo en los dos estados de ansiedad, así como por entrenar más días a la semana y llevar más años entrenando, por el menor nivel de la competición en la que participa y por tener apoyo de un entrenador. Los resultados confirmaron la hipótesis en cuanto a los niveles de ansiedad y autoconfianza pero, sin embargo, se obtuvieron resultados inesperados en cuanto a la predicción de las mismas que posteriormente se discuten.
\end{abstract}

Palabra clave: adultos, ansiedad, entrenamiento, rendimiento, motivación.

Abstract: In recent years Spain has seen a very significant increase in adults over 35 who often train and compete at all levels in athletics tracks. They are master athletes and, above them, there is no nationwide study and very few internationally there. Therefore, the objective of this research is on the one hand, know their training habits, athletic history and their anxiety level, analyzing gender differences, and secondly, obtain predictive models of anxiety based on their training habits and athletic history. We start from the assumption that, in the Spanish veteran athletes, in both sexes, there should be a moderate pre-competitive anxiety (cognitive and somatic) and high self-confidence, and anxiety will be predict by a high score in the other state of anxiety, by scoring low in self-confidence as well as to train fewer days a week and take less years training, for the highest level of competition in which they participates and not having support from a coach. By contrast, self-confidence can be predicted by low rate in the two states of anxiety, by training more days a week and have more years training, by the lower level of the competition in which it participates and for having supported a coach. The results confirmed the hypothesis regarding levels of anxiety and self-confidence but, however, unexpected results were obtained regarding the prediction thereof which are discussed later.

Key words: adults, anxiety, training, performance, motivation.

1. Introducción

Anshel et al. (1991) definieron la ansiedad como un sentimiento subjetivo de aprensión o amenaza percibida, a veces acompañada por un incremento de la activación fisiológica. Jaenes (2000), en función de la estabilidad de la ansiedad, distinguía entre ansiedad rasgo (ser) como la tendencia personal a tener ansiedad en la mayoría de situaciones de la vida cotidiana, y ansiedad estado (estar) como la manifestada por una persona en una situación concreta.

Desde una aproximación multidimensional, Martens, Burton, Vealey, Bump y Smith (1990) diferenciaron en la ansiedad precompetitiva tres componentes en función de a qué nivel se manifestaba. El primero es una reacción cognitiva, el componente mental de la ansiedad y está causado por expectativas negativas sobre el éxito o por autoevaluación negativa. El segundo lo explicaron como una reacción fisiológica o somática, los elementos afectivos y fisiológicos de la experiencia de ansiedad derivados directamente de la activación autonómica. Por último, al tercer componente de la teoría multidimensional de la ansiedad lo llamaron autoconfianza, que no es una medida directa de ansiedad, pero su ausencia sugiere que el deportista puede experimentar ansiedad cognitiva.

La ansiedad es un constructo psicológico ampliamente estudiado en el campo de la conducta humana en general y de la psicología del deporte en particular. El motivo son sus repercusiones posteriores en el rendimiento de los deportistas, de manera que, según estos controlen sus síntomas, puede aumentarles o disminuirles el rendimiento (Smith, 1989). Jones (1991) matizaba que, en función de si un deportista percibe como favorable o desfavorable los síntomas de la ansiedad

Fecha recepción: 21-10-12 - Fecha envío revisores: 21-10-12 - Fecha de aceptación: 01-12-12 Correspondencia: Francisco Ruiz Juan

Calle Cabo Vidio, 27

30730, San Javier (Murcia)

E-mail: pacoruizjuan @ gmail.com precompetitiva, por ejemplo los síntomas de la ansiedad somática, puede beneficiarle o perjudicarle en su ejecución o rendimiento posterior. Así, aunque en la literatura hay trabajos en los que se concluye que la ansiedad precompetitiva facilita el rendimiento (Jones \& Swain, 1995), en la mayoría de las investigaciones se concluye que la ansiedad tiene efectos negativos no sólo en el rendimiento, si no en el disfrute de la participación deportiva y en el bienestar físico (Scanlan, Babkes \& Scanlan, 2005; Scanlan \& Lewthwaite, 1986; Smith, Smoll, \& Wiechman, 1998).

En los últimos años se ha debatido sobre cuál es el peso que tiene uno u otro tipo de ansiedad en la realización de la tarea deportiva. Furst y Tenembaum (1984) encontraron una relación curvilínea de U invertida entre ansiedad y rendimiento, concluyendo además que los deportistas de equipo mostraban menor ansiedad que los individuales. Posteriormente, Gould, Petlichkoff, Simon y Vevera (1987) concluyeron que existía esa relación curvilínea de U invertida concretamente entre ansiedad somática y rendimiento, pero no hallaron datos significativos respecto a la ansiedad cognitiva y la autoconfianza. Burton (1988) también encontró la misma relación curvilínea entre rendimiento y ansiedad somática, pero además obtuvo una relación negativa entre la ansiedad cognitiva y el rendimiento, y positiva entre éste y la autoconfianza.

Es necesario añadir que el ambiente en el que se encuentra compitiendo el deportista (Arbinaga \& Caracuel, 2005), el tipo y características de la prueba a la que se enfrenta (Jaenes, 2000), así como el tipo de deporte (Martens, et al., 1990), la experiencia en competición (Mellalieu, Hanton \& O'Brien, 2004) e incluso la percepción del deportista de ir o no adecuadamente entrenado a competir (Gutiérrez, Estévez, García \& Pérez, 1997), pueden influir en el grado en que se manifiestan los síntomas de la ansiedad precompetitiva en un deportista.

De la misma manera, también han sido investigadas las predicciones de la ansiedad en función de la percepción del éxito en el deporte, de forma que una mayor ansiedad fue observada en deportistas con una alta orientación al ego y poca autoconfianza en símismos (Voigh, Callaghan 
\& Ryska, 2000). Por su parte, Cervelló, Santos-Rosa, Jiménez, Nerea y García (2002) matizaron que la orientación disposicional al ego predice significativamente de manera negativa la ansiedad cognitiva, mientras que la orientación disposicional a la tarea predice significativa y positivamente la autoconfianza.

Sin embargo, según datos de la Real Federación Española de Atletismo (RFEA, 2012) en España existe una población de deportistas que en los últimos quince años ha incrementado significativamente su número, los atletas veteranos (mayores de 35 años) que entrenan y compiten en pista de atletismo, de la que poco o nada se sabe ni a nivel psicológico, ni a nivel descriptivo.

Recientemente, en esta población Ruiz-Juan y Zarauz (2012a) analizaron su grado de autodeterminación en función, no de otros constructos psicológicos, sino de una serie de hábitos de entrenamiento y de su historial atlético. Por un lado, concluyeron que poseían elevados niveles de motivación intrínseca, moderados de extrínseca y casi nulos de amotivación. Por otro, acabaron obteniendo unos sólidos modelos de estos tres niveles de autodeterminación en función de las variables de entrenamiento e historial atlético mencionadas.

En deportistas adultos españoles hay pocos estudios de estas características. Así, Ruiz-Juan y Zarauz (2012b), en un estudio sobre la adicción negativa a correr de los maratonianos españoles, obtuvieron que la diferencia entre los maratonianos con mayores valores de adicción negativa a correr (no deseable) y los de menor adicción negativa a correr eran, siendo varones, hacer tanto un número significativamente mayor de kilómetros y días de entrenamiento a la semana como de horas por entrenamiento, preocuparse algo más por buscarse un entrenador y tener una mejor marca cercana a las 3 horas y media en los 6-7 maratones que han terminado, frente a una menor preocupación por tener entrenador y ser su primer maratón, en los corredores con puntuaciones mínimas en adicción negativa a correr. Lo que no se esperaron fue que entre las mujeres no hubiese diferencias significativas.

Por ello, el objetivo de la presente investigación pasa por arrojar más luz sobre los interrogantes aún abiertos en esta población de atletas veteranos españoles. Así, trataremos de conocer sus niveles de ansiedad precompetitiva y obtener unos modelos predictores de la misma suficientemente sólidos en función de una serie de variables de entrenamiento y de su historial atlético.

Se parte de la hipótesis de que, en los atletas veteranos españoles, en ambos sexos, debe existir una moderada ansiedad precompetitiva (cognitiva y somática) y alta autoconfianza, y de que se van a obtener sólidos modelos predictores de dichos constructos, con algunas diferencias entre sexos. Así, como sugiere la literatura consultada, la ansiedad se podrá predecir por puntuar alto en el otro estado de ansiedad, por puntuar bajo en autoconfianza, así como por entrenar menos días a la semana y llevar menos años entrenando, por el mayor nivel de la competición en la que participa y por no tener apoyo de un entrenador. Por el contrario, la autoconfianza se podrá predecir por puntuar bajo en los dos estados de ansiedad, así como por entrenar más días a la semana y llevar más años entrenando, por el menor nivel de la competición en la que participa y por tener apoyo de un entrenador.

\section{Método}

\subsection{Participantes}

Se partió del total de atletas veteranos de pista participantes en diversas competiciones organizadas específicamente para ellos de nivel provincial, autonómico y nacional en el territorio Español del año 2011. Según datos de la RFEA (2011), hubo 5704 licencias federativas de atletas veteranos de pista, de los cuales 4727 son hombres y 977 son mujeres.

Se obtuvo una muestra aleatoria de 401 atletas veteranos de pista, de los cuales 330 son hombres (82.3\%) con rango de edad de 35 a 75 años $(M=47.98 ; D T=9.14)$ y 71 mujeres $(17.7 \%)$ con rango de edad de 35 a 69 años $(M=45.78 ; D T=10.25)$. Por tanto, se tiene una muestra representativa con un error del $\pm 4.82 \%$, a un intervalo de confianza del $95.5 \%$.

\subsection{Procedimiento}

Para obtener una muestra lo más amplia y dispersa geográficamente en el territorio español, se pidió colaboración al responsable nacional de atletas veteranos para colgar un enlace en la sección de Veteranos de la Web de la RFEA (http://www.rfea.es/veteranos/veteranos.asp) y así todos los veteranos que visitan la página puedan rellenar el cuestionario telemáticamente. Igualmente, al webmaster del principal foro de atletismo de España (http://www.elatleta.com/foro/forum.php) se le pidió colocar en la sección de atletas veteranos.

En la portada de la Web (http://www.retos.org/2encuesta/ inicio.html) se informaba del objetivo del estudio, de la voluntariedad, absoluta confidencialidad de las respuestas y manejo de los datos, de que no había respuestas correctas o incorrectas, y se les solicitó que contestaran con la máxima sinceridad y honestidad. Este trabajo posee informe favorable de la Comisión de Bioética de la Universidad de Murcia.

\subsection{Instrumento}

$\varnothing$ Inventario de Ansiedad Competitiva-2 Revisado (CSAI-2R); versión española de Andrade et al. (2007) de Revised Competitive State Anxiety Inventory-2 (CSAI-2R) de Cox et al. (2003). Tiene 3 subescalas: ansiedad cognitiva, ansiedad somática y autoconfianza. La primera y tercera contienen 5 ítems puntuables de 1 (nada) a 4 (mucho) con puntuación total entre 5 y 20. La segunda contiene 6 ítems, con puntuación total entre 6 y 24 . Diversos estudios han mostrado su validez exploratoria, confirmatoria y su fiabilidad (Andrade, et al., 2007).

$\varnothing$ Hábitos de entrenamiento e historial atlético. Los encuestados respondieron a: ¿Tiene entrenador?, ¿Cuánto tiempo entrena normalmente al día?, ¿Cuántos días entrena a la semana?, ¿Cuántos años lleva entrenando?, ¿En cuántas competiciones participa al año?, ¿A qué nivel ha llegado a competir en su mejor especialidad? ¿Cuál es su especialidad atlética habitual?

\subsection{Análisis de los datos}

Los análisis de ítems, homogeneidad, correlación entre las subescalas (coeficiente de Pearson), consistencia interna (alfa de Cronbach), diferencias de medias por sexo (t Student), correlaciones entre todas las dimensiones de las escalas y regresión lineal multivariante, se realizaron con el SPSS 20.0. La estructura factorial se examinó con un análisis factorial confirmatorio (AFC) con el AMOS 18.0.

\section{Resultados}

\subsection{Propiedades psicométricas del instrumento}

En el análisis de ítems de la escala, ningún ítem fue eliminado al cumplir los requisitos establecidos (valore».30 en el coeficiente de correlación corregido ítem-total, desviación típica $>1$ y todas las opciones de respuesta fueron usadas). El análisis de la homogeneidad indicó inexistencia de solapamientos de ítems entre las dimensiones teóricas utilizadas en la escala. Se calcularon índices de asimetría y curtosis siendo, en general, próximos a cero y $<2.0$, tal y como recomiendan Bollen y Long (1994), lo que indica semejanza con curva normal de forma univariada.

La validez factorial se examinó utilizando AFC. Se utilizó estimación de máxima verosimilitud, teniendo que recurrir a la técnica «bootstrapping»y al procedimiento de máxima verosimilitud $(M L)$, procedimiento de estimación de modelos de ecuaciones estructurales que asume distribución normal de datos y escala continua de ellos, pues el coeficiente Mardia (siempre >2) indica falta de normalidad multivariada en los datos con lo que se violaba una regla básica del AFC. El ajuste del modelo fue evaluado con combinación de índices de ajuste absolutos y relativos. El modelo presenta valores correctos y aceptable bondad de ajuste del modelo original (Hoyle, 1995; Hu \& Bentler, 1999; Kline, 1998) como manifiestan los resultados obtenidos: $\div 2 / g l=2.18, G F I=.96$, $C F I=.97, I F I=.96, T L I=.95, R M S E A=.031, S R M R=.025$. Los coeficientes estandarizados de relación de la variable latente con cada uno de los ítems, oscilaron entre .69 y .85 . 
Como se predijo, tanto los veteranos como las veteranas del presente estudio han obtenido puntuaciones altas en autoconfianza y moderadas en ansiedad cognitiva y somática. Muy probablemente sea debido tanto a la madurez de la edad de esta población, como al alto grado de conocimiento y experiencia de su especialidad deportiva, tanto en entrenamiento como en competiciones, como se desprende del análisis de los datos de sus hábitos de entrenamiento e historial atlético (Tabla 1).

Aun teniendo valores moderados en ansiedad ambos sexos, lo que no se predijo fue que las mujeres puntuasen en ansiedad somática significativamente más alto que los varones. De la misma manera, obteniendo puntuaciones altas los dos sexos, lo que tampoco se predijo fue que los hombres puntuasen significativamente más alto que las mujeres en autoconfianza. Todo esto puede estar debido precisamente a las aludidas diferencias en sus hábitos de entrenamiento e historial atlético, puesto que son precisamente ellas las que tienen un número significativamente menor que ellos de días de entrenamiento por semana y de años entrenando.

Del análisis de las correlaciones y modelos predictores de la ansiedad y la autoconfianza en hombres, el mayor número de años entrenando vuelve a mostrarse como una variable determinante a la hora de moderar la ansiedad somática y elevar la autoconfianza. Sin embargo, el resto de variables con las que se hipotetizó iban a ser determinantes en el grado en que se manifestaban los síntomas de la ansiedad, resultaron no serlo. La única explicación posible es que a los atletas veteranos, debido a la madurez que les confiere su edad, la cantidad de años que llevan practicando su especialidad atlética, el elevado número de veces que han competido en la misma y su alto grado de dominio en ella, les confiere la suficiente autoconfianza como para no generarles ansiedad el hecho de no tener entrenador, que la competición sea de máximo nivel o haber podido entrenar menos días a la semana para preparar una competición, como puede sucederle a los atletas más jóvenes y con menor experiencia (Arbinaga \& Caracuel, 2005; Gutiérrez, etal., 1997; Mellalieu, et al., 2004).

Sin embargo, aunque se contaba con diferencias entre sexos en la hipótesis del presente estudio, no se predijo que a las veteranas, ni en las correlaciones ni en los modelos regresivos, ninguna variable de entrenamiento o historial atlético resultaron determinantes a la hora de influir en su ansiedad o autoconfianza. Ello no hace sino ahondar en la necesidad de seguir estudiando este constructo psicológico en esta población, incluyendo nuevas variables que puedan arrojar luz sobre los interrogantes aun por desvelar.

\section{Referencias}

Andrade, E. M., Lois, G., \& Arce, C. (2007). Propiedades psicométricas de la versión española del Inventario de Ansiedad Competitiva CSAI2R en deportistas. Psicothema, 19(1), 150-155.

Anshel, M., Freedson, P., Hamill, J., Haywood, K., Horvat, M., \& Plowman, S. (1991). Dictionary of the sport and exercise sciences. Champaign, IL: Human Kinetics.

Arbinaga Ibarzábal, F, \& Caracuel Tubío, J. C. (2005). Precompetición y ansiedad en fisicoculturistas. Revista de Psicología del Deporte, 14(2), 195-208.

Bollen, K. A., \& Long, J. (1994). Testing structural equation models. Newbury Park, CA: Sage.

Burton, D. (1988). Do anxious swimmers swim slower? reexamining the elusive anxiety-performance relationship. Journal of Sport and Exercise Psychology, 10, 45-61.

Cervelló, E., Santos-Rosa, F. J., Jiménez, R., Nerea, A., \& García, T. (2002). Motivación y ansiedad en jugadores de tenis. Motricidad, 9 , 141-161.

Cox, R. H., Martens, M. P., \& Russell W. D. (2003). Measuring anxiety in athletics: The revised Competitive State Anxiety Inventory-2. Journal of Sport and Exercise Psychology, 25, 519-533.

Furst, D. M., \& Tenembaum, G. (1984). Sport performance and various state anxiety components: A cross sectional study. Netanya: Wingate Institute.
Gould, D., Petlichkoff, L., Simon, J., \& Vevera, M. (1987). The relationship between Competitive State Anxiety Inventory-2 subscale scores and pistol shooting performance. Journal of Sport Psychology, 9, 3342.

Gutiérrez Calvo, M., Estévez, A., García Pérez, J., \& Pérez Hernández, H. (1997). Ansiedad y rendimiento atlético en condiciones de estrés: efectos moduladores de la práctica. Revista de Psicología del Deporte, 6(2), 27-46.

Hoyle, R. H. (1995). Structural equation modeling: Concepts, issues, and applications. Thousand Oaks, CA: Sage.

Hu, L., \& Bentler, P. M. (1999). Cutoff criteria for fit indexes in covariance structure analysis: Conventional criteria versus new alternatives. Structural Equation Modeling, 6, 1-55.

Jaenes Sánchez, J. C. (2000). Estado emocional y conducta deportiva: Ansiedad competitiva en corredores de maratón. Tesis Doctoral. Universidad de Sevilla.

Jones, J. G. (1991). Recent developments and current issues in competitive state anxiety research. The Sport Psychologist, 4, 152-155.

Jones, G., \& Swain, A. (1995). Predisposition to experience debilitative and facilitative anxiety in elite and non-elite performers. The Sport Psychologist, 9, 201-211.

Kline, R. B. (1998). Principles and practice of structural equation modeling. New York: Guilford.

Martens, R., Burton, D., Vealey, R. S., Bump, L. A., \& Smith, D. E. (1990). The Competitive State Anxiety Inventory-2 (CSAI-2). En R. Martens, R. S. Vealey y D. Burton (Eds), Competitive Anxiety in Sport. Champaign IL: Human Kinetics.

Mellalieu, S., Hanton, S., \& O’Brien, M. (2004). Intensity and direction of competitive anxiety as a function of sport type and experience. Scandinavian Journal of Medicine and Science in Sports, 14, 326334.

Real Federación Española de Atletismo (2012). Comparativa histórica de Licencias de Veteranos. Recuperado de http://www.rfea.es/veteranos/ licencias.asp

Ruiz-Juan, F., \& Zarauz,A. (2011). Validación de la versión española de las Motivations of Marathoners Scales (MOMS). Revista Latinoamericana de Psicología, 43(1), 139-156.

Ruiz-Juan, F., \& Zarauz, A. (2012a). Predictor variables of motivation in Spanish master athletes. Journal of Human Sport and Exercise, $7(3), 617-628$.

Ruiz-Juan, F., \& Zarauz, A. (2012b). Variables que hacen adicto negativamente a correr al maratoniano español. Retos, 21, 38-42.

Scanlan, T. K., Babkes, M. L., \& Scanlan, L. A. (2005). Participation in sport: A developmental glimpse at emotion. En J. L. Mahoney, R. W. Larson \& J. S. Eccles (Eds.). Organized activities as contexts of development: Extracurricular activities, after school, and community programs. (pp. 275-309). Mahwah, NJ: Erlbaum.

Scanlan, T. K., \& Lewthwaite, R. (1986). Social psychological aspects of competition for male youth sport participants: IV. Predictors of enjoyment. Journal of Sport Psychology, 8, 25-35.

Smith, R. (1989). Athletic stress and burnout: conceptual models and intervention strategies. En D. Hackfort y C. D. Spielberger (eds.): Anxiety in sports: An international perspective (pp. 183-201). Nueva York, NY: Hemisphere.

Smith, R. E., Smoll, F. L., \& Wiechman, S. (1998). Measurement of trait anxiety in sport. En J. L. Duda (Ed.), Advances in sport and exercise psychology measurement. Morgantown, WV: Fitness Information Technology.

Voight, M. R., Callaghan, J. L., \& Ryska, T. A. (2000). Relationship between goal orientations, self-confidence and multidimensional trait anxiety among Mexican-American female youth athletes. Journal of Sport Behavior, 23(3), 271-288.

Zarauz, A., \& Ruiz-Juan, F. (2011). Compromiso y adicción negativa al entrenamiento y competición de los maratonianos. Revista Internacional de Medicina y Ciencias de la Actividad Física y el Deporte, 11(44), 817-834.

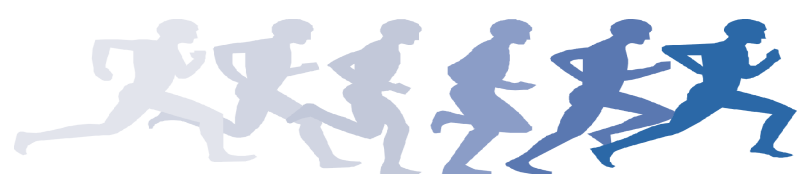

